Supporting Information for:

\title{
Vertically Integrated Multiple Nanowire Field Effect
}

\section{Transistor}

Byung-Hyun Lee, ${ }^{1,2}$ Min-Ho Kang, ${ }^{3}$ Dae-Chul Ahn, ${ }^{1}$ Jun-Young Park, ${ }^{1}$ Tewook Bang, ${ }^{1}$

Seung-Bae Jeon, ${ }^{1}$ Jae Hur, ${ }^{1}$ DongIl Lee, ${ }^{1}$ and Yang-Kyu Choi ${ }^{1 *}$

${ }^{1}$ Department of Electrical Engineering, Korea Advanced Institute of Science and Technology (KAIST), 291 Daehak-ro, Yuseong-gu, Daejeon 305-701, Republic of Korea

${ }^{2}$ Department of Memory Business, Samsung Electronics, San \#16 Banwol-Dong, Hwasung-City, Gyeonggi-Do 445-701, Republic of Korea

${ }^{3}$ Department of Nano-process, National Nanofab Center (NNFC), 291 Daehak-ro, Yuseong-gu, Daejeon 305-701, Republic of Korea

Byung-Hyun Lee: bhlee@nobelab.kaist.ac.kr

Min-Ho Kang:kmh@nnfc.re.kr

Dae-Chul Ahn:dcahn@nobelab.kaist.ac.kr

Jun-Young Park: jypark@nobelab.kaist.ac.kr 
Tewook Bang: twbang@nobelab.kaist.ac.kr

Seung-Bae Jeon: sbjeon@nobelab.kaist.ac.kr

Hur Jae: jhur@nobelab.kaist.ac.kr

DongIl Lee: dilee@nobelab.kaist.ac.kr

Yang-Kyu Choi: ykchoi@ee.kaist.ac.kr

* Address correspondence to ykchoi@ee.kaist.ac.kr. 


\title{
Table of contents
}

\author{
1. Fabrication process
}

2. Experimental equipment

3. Nanowire FETs with performance booster

4. GIDL in the VM-FET

5. VM-FET with laterally parallel stacks of the nanowire 


\section{Supporting Information 1: Fabrication process}

To fabricate the vertically integrated multi-nanowire (multi-NW)-based field-effecttransistor (FET), a boron-doped (100) bulk-silicon (Si) wafer was used as a substrate. Boron ions were implanted on a bulk Si to block the leakage path below the NW channel, in a process known as channel stop implantation. High-density plasma (HDP) oxide, which acts as a hard mask to etch $\mathrm{Si}$ for the formation of vertically integrated multi-NWs, was deposited using plasma enhanced chemical vapor deposition (PECVD). Photolithography using a krypton fluoride (KrF) laser was conducted on a HDP oxide hard mask, after which the hard mask was etched to pattern the channel region. In order to form the vertically integrated multi-NW structure, iterative deep reactive-ion etching (RIE) was done under the optimum process conditions;

1. $\mathrm{C}_{4} \mathrm{~F}_{8}$ passivation: $300 \mathrm{sccm} / 6 \mathrm{~m}$ Torr / plasma power $3000 \mathrm{~W} /$ each $1 \mathrm{~s} /$ chuck temperature: $-5^{\circ} \mathrm{C}$ )

2. $\mathrm{SF}_{6}$ etching process: $400 \mathrm{sccm} / 5 \mathrm{~m}$ Torr / plasma power $3000 \mathrm{~W} / 2.4 \sim 3 \mathrm{~s} /$ chuck temperature: $-5^{\circ} \mathrm{C}$ )

As shown in Figure 1a, one cycle of the process consists of $\mathrm{C}_{4} \mathrm{~F}_{8}$-based polymer passivation and $\mathrm{SF}_{6}$-based isotropic dry etching. The iteration of the cycle allowed the formation of a number of NWs with a uniform profile without the additional carving process: subsequent oxidation and wet etching. Figure S1 shows the failed profile under a non-optimized condition. The vertically integrated multi-NW pattern created by the optimized one-route all-dry etching process (ORADEP) is shown in Figure S2.

To isolate the transistors, tetraethyl orthosilicate (TEOS) as an inter-layer dielectric (ILD) was deposited using low pressure chemical vapor deposition (LPCVD), followed by a chemical mechanical polishing (CMP) process for planarization. The sequential pattering process was 
done to form a gate-all-around (GAA) structure. First, photolithography process was conducted to form the trench hole pattern on both sides of the NW, after which the partial dry etch process was conducted. Subsequently, a wet etch process to merge the two trench holes located on the both sides of NW was applied, resulting in the release of the NWs from the ILD oxide through the trench hole with the width of about $600 \mathrm{~nm}$. The dry and wet etch rates should be thoroughly controlled such that the ILD oxide remains between the bottom NW and the Si substrate. The remaining oxide plays a crucial role in blocking leakage paths from the source (S) to the drain (D), similar to the channel stop implantation concept. Gate oxide of $5 \mathrm{~nm}$-thick was formed for a metal-oxide-semiconductor FET (MOSFET) by means of thermal wet oxidation. In this case, in order to form the silicon-oxide-nitride-oxide-silicon (SONOS) structure for nonvolatile memory applications, the O/N/O structure was formed using thermal oxidation followed by LPCVD. After the formation of the gate dielectric for the MOSFET and SONOS memory, a doped poly-Si for gate was deposited using LPCVD, followed by a CMP process for planarization, which results in the formation of the GAA configuration. In order to protect the actual channel region during the self-aligned S/D implantation process, oxide and nitride were sequentially deposited on the planarized poly-Si layer as an implant stopper, and then they were patterned by a conventional photolithography and dry etching process. Phosphorous ions were implanted to form S/D electrode. Self-aligned S/D implantation was carried out while the exposed gate blocked the dopants as the implant stopper. Thus excepting the region covered by the exposed gate, all other areas were heavily doped. Stable switching operation of the VM-FET in Figure 4 and Figure S3 demonstrates the stability and acceptable variability arisen from the fabrication process. Finally, the annealing process for activation of the dopant and forming gas annealing (FGA) were done to improve the $\mathrm{Si} / \mathrm{SiO}_{2}$ interface characteristics. A schematic of the fabricated 
device showing the dimension of the device parameters is illustrated in Figure S3. In addition, the electrical characteristics of the fabricated VM-FET with various gate lengths are shown in Figure S4.

(a)

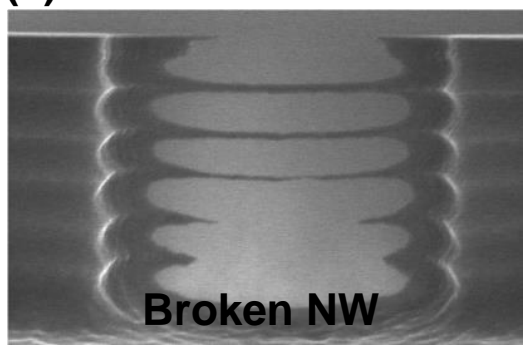

(b)

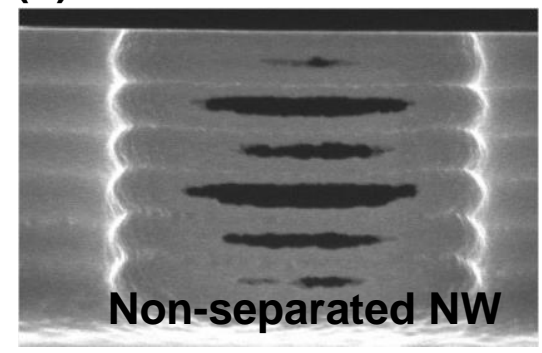

(c)

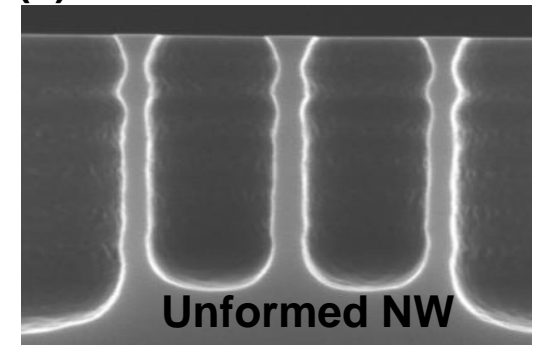

Figure S1. SEM image of a failed multi-NW created by the non-optimized process. (a) Crosssectional SEM image of the broken NW. The profile was caused by recessive SF6-based isotropic etching or a lack of sufficient $\mathrm{C}_{4} \mathrm{~F}_{8}$-based polymer passivation. (b) Cross-sectional SEM image of the non-separated NW, which was mainly attributed to deficient etching. (c) Crosssectional SEM image of an unformed NW. The recessive polymer passivation process hinders the stable formation of the scallop pattern.
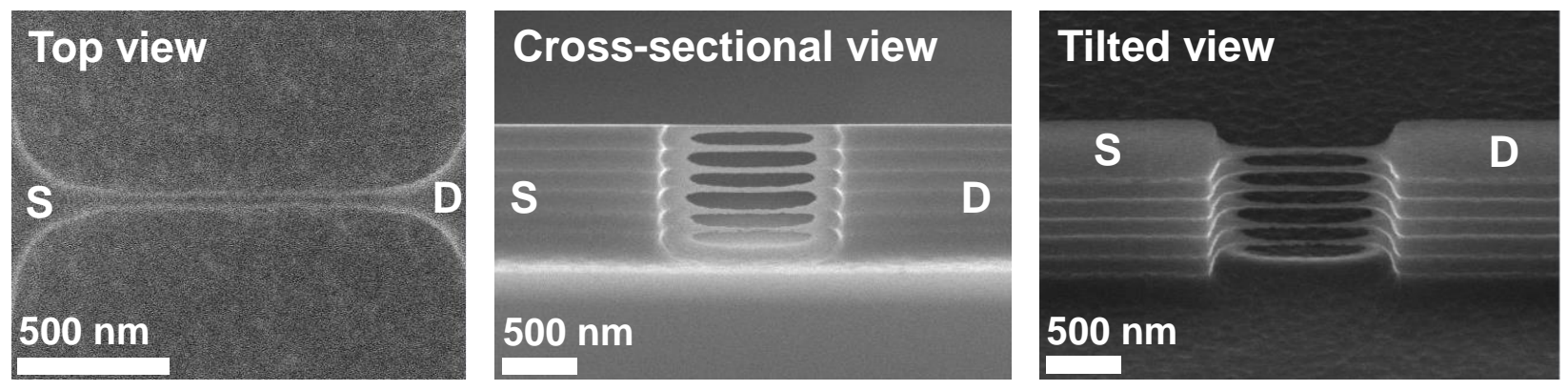

Figure S2. SEM image of the multi-NW structure created with the optimized process condition.

(a) Top-view SEM image. (b) Cross-sectional SEM image. (c) Tilted SEM image. 


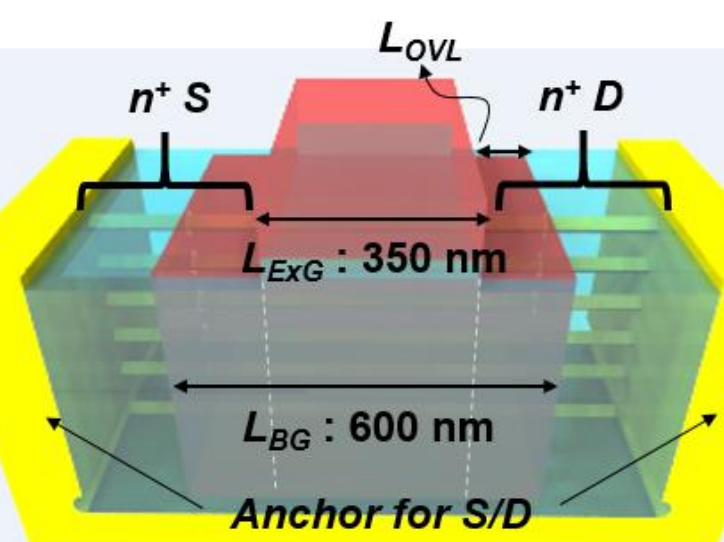

Figure S3. A schematic of the fabricated VM-FET showing the various device parameters. In the schematic, $\boldsymbol{L}_{\boldsymbol{E} \boldsymbol{x} \boldsymbol{G}}$ and $\boldsymbol{L}_{\boldsymbol{B} \boldsymbol{G}}$ represent a length of the exposed gate and buried gate, respectively. And $\boldsymbol{L}_{\boldsymbol{O} V \boldsymbol{L}}$ is the overlap length of gate-to-S/D.

(a)

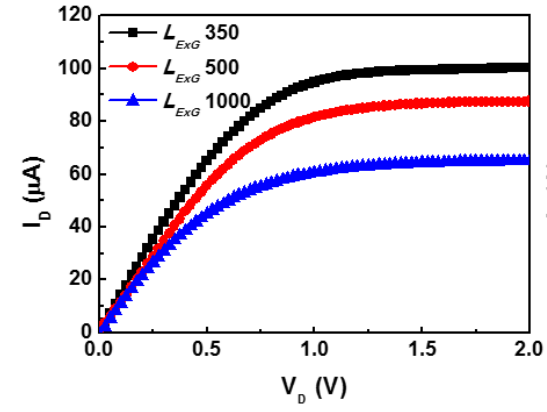

(b)

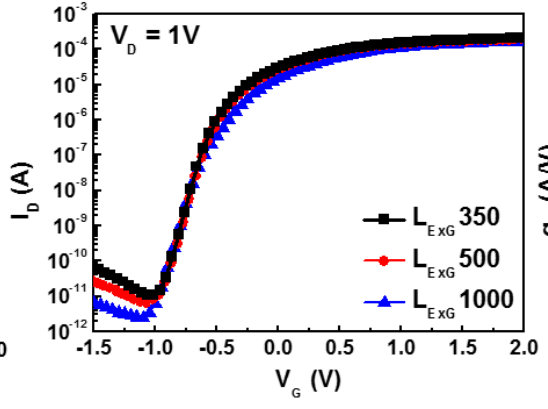

(c)

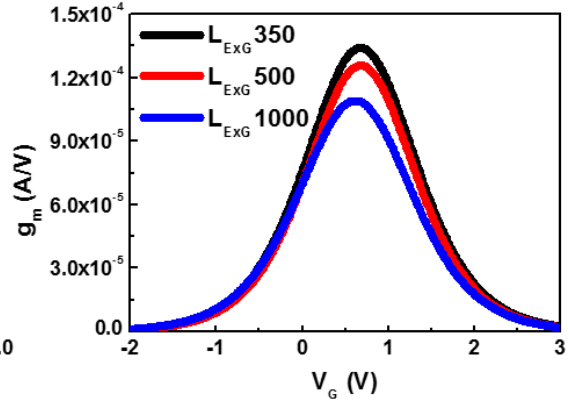

Figure S4. Electrical characteristic of the fabricated VM-FET with various gate length. (a) $I_{D^{-}} V_{D}$ characteristics with various exposed gate lengths. (b) $I_{D^{-}} V_{G}$ characteristics with various exposed gate lengths. (c) $g_{m}-V_{G}$ characteristics with various exposed gate lengths. There is no notable degradation of the device with respect to the variation of the gate length, supporting the acceptable process variability. 


\section{Supporting Information 2: Experimental equipment}

All fabrication processes were done in a clean FAB (National Nanofab Center, NNFC in Korea). The equipment list for main fabrication processes is as follows; Vendor, Model

1. Photolithography process : Nikon, NSR-S203B (KrF Scanner)

2. Oxide dry etching process : Lam Research, TCP9400DFM

3. One-route all-dry etching process for $\mathrm{Si}$ : Alcatel, AMS2000

4. Thermal oxidation and LPCVD process : CENTROTHERM, E1200

5. Deposition of HDP oxide: Novellus, Concept II -SPEED

6. CMP process : Doosan DND, UNIPLA ${ }^{\mathrm{TM}} 231$

7. Implantation process : DIM20, Excelis

8. RTA and FGA process : Metron, AG Heatpulse 8800

9. Wet etching and cleaning process : HIT, NXWET

10. Monitoring thickness of the layers : KLA-Tencor, SFX100

11. Monitoring critical dimension of each pattern : Hitachi, S-9260A

SEM (S-4800) was used to analyze cross-sectional images of the multi-NW structure after the deep RIE process. In order to prepare the sample for transmission electron microscopy (TEM), a focused ion beam (FIB, Helios Nanolab) process was employed after the passivation of the device using plasma-enhanced nitride and platinum. The cross-sectional TEM image of the fabricated device was obtained from using high-resolution TEM (JEM-ARM200F) with a fast Fourier transform (FFT) analysis to confirm the single crystal also utilized in the same equipment. The analysis of the components and a clear definition of each layer were achieved by energy-dispersive X-ray spectroscopy (EDS, Quantax 400). Secondary ion mass spectroscopy (SIMS, IMS7f) was used to determine the proper implantation energy via an analysis of the 
doping profile with the depth. All electrical measurements were carried out in the absence of device encapsulation. The current-voltage (I-V) characteristics were measured using a HP4156 semiconductor parameter analyzer. The memory function and reliability results were obtained from a HP4156 and a pulse generator (Agilent 81110A), respectively. 


\section{Supporting Information 3: Nanowire FETs with performance booster}

The performance boosters such as strained-silicon, high-k and metal gate (HKMG), and sicilicide process for $\mathrm{S} / \mathrm{D}$ formation are effective for the enhancement of the device performance. The research results employing such performance boosters are reviewed in Table S1. This summary would suggest an insight for enhancing the performance of further vertically integrated multi-NW FET (VM-FET).

\begin{tabular}{|c|c|c|c|c|c|}
\hline Ref & 1 & 2 & 3 & 4 & 5 \\
\hline Substrate & SOI & sSOI & SOI & SOI & SGOI \\
\hline Channel & strained-Si & strained-Si & strained-Si & strained-Si & strained-Si \\
\hline Structure & $\begin{array}{l}\text { Tri-gate } \\
\text { Nanowire }\end{array}$ & $\begin{array}{c}\Omega \text {-gate } \\
\text { Nanowire }\end{array}$ & $\begin{array}{l}\text { Tri-gate } \\
\text { Nanowire }\end{array}$ & $\begin{array}{l}\text { Tri-gate } \\
\text { Nanowire }\end{array}$ & $\begin{array}{c}\Omega \text {-gate } \\
\text { Nanowire }\end{array}$ \\
\hline $\begin{array}{l}\text { Strained } \\
\text { technology }\end{array}$ & $\begin{array}{c}\text { Stress } \\
\text { memorization tech } \\
\text { (SMT) }\end{array}$ & $\begin{array}{c}\text { Si:Ge } \\
\text { (Raised S/D) }\end{array}$ & $\begin{array}{l}\mathrm{Ni}(\mathrm{Al}) \mathrm{Si}: \mathrm{C} \& \\
\mathrm{ZnS}_{-} \mathrm{SiO}_{2}\end{array}$ & $\begin{array}{c}\text { S/D silicide \& } \\
\text { GeTe }\end{array}$ & $\begin{array}{c}\mathrm{Si}: \mathrm{Ge} \\
\text { (Raised S/D) }\end{array}$ \\
\hline S/D formation & Implantation & $\begin{array}{l}\text { Raised Si:Ge \& } \\
\text { Implantation }\end{array}$ & Implantation & $\begin{array}{c}\text { Silicide \& } \\
\text { Implantation }\end{array}$ & $\begin{array}{l}\text { Raised Si:Ge \& } \\
\text { Implantation }\end{array}$ \\
\hline HKMG & X & $\mathrm{O}$ & $x$ & Only MG & 0 \\
\hline $\begin{array}{l}\text { Dimension } \\
\text { (L/W) }\end{array}$ & $\begin{array}{l}14 \mathrm{~nm} / \\
11 \mathrm{~nm}\end{array}$ & $\begin{array}{l}10 \mathrm{~nm} / \\
14 \mathrm{~nm}\end{array}$ & $45 \mathrm{~nm} / 55 \mathrm{~nm}$ & $\begin{array}{l}35 \mathrm{~nm} / \\
45 \mathrm{~nm}\end{array}$ & $\begin{array}{c}15 \mathrm{~nm} / \\
7 \mathrm{~nm}\end{array}$ \\
\hline$I_{D, o n}$ & $\begin{array}{c}24(\mu A) \\
@ V_{D}=1 V \\
\& V_{O D}=1 V\end{array}$ & $\begin{array}{c}80(\mu A) \\
@ V_{D}=0.9 V \\
\& V_{O D}=1 V\end{array}$ & $\begin{array}{c}60(\mu \mathrm{A}) \\
@ \mathrm{~V}_{\mathrm{D}}=1.2 \mathrm{~V} \\
\& \mathrm{~V}_{\mathrm{OD}}=1 \mathrm{~V}\end{array}$ & $\begin{array}{c}22(\mu A) \\
@ V_{D}=-1.2 \mathrm{~V} \& V_{O D} \\
=1 \mathrm{~V}\end{array}$ & $\begin{array}{c}30(\mu A) \\
@ V_{D}=1 V \\
\& V_{O D}=1 V\end{array}$ \\
\hline$I_{D, \text { off }}$ & $\begin{array}{l}1.2 \times 10^{-13}(A) \\
@ V_{D}=1 V\end{array}$ & $\begin{array}{l}2.8 \times 10^{-11}(A) \\
@ V_{D}=0.9 \mathrm{~V}\end{array}$ & $\begin{array}{l}7.5 \times 10^{-11}(A) \\
@ V_{D}=1.2 \mathrm{~V}\end{array}$ & $\begin{array}{c}8 \times 10^{-11}(A) \\
@ V_{D}=-1.2 \mathrm{~V}\end{array}$ & $\begin{array}{l}1 \times 10^{-11}(A) \\
@ V_{D}=1 V\end{array}$ \\
\hline \multirow[t]{2}{*}{ Type } & nMOS & nMOS & nMOS & pMOS & pMOS \\
\hline & \multicolumn{4}{|c|}{$\begin{array}{c}\text { Width normalized factor : perimeter }\left(W_{N W}+2 H_{N W}\right) \\
\cdot \mathrm{V}_{\mathrm{OD}}=\mathrm{V}_{\mathrm{G}}-\mathrm{V}_{\mathrm{TH}} \text {, overdrive voltage }\end{array}$} & \\
\hline
\end{tabular}

Table S1. Research results of the nanowire-FET employing state-of-the-art technology

\section{Reference}

1. Saitoh, M.; Ota, K.; Tanaka, C.; Uchida, K.; Numata, T. VLSI Tech. Symp. 2012, 11-12.

2. Barraud, S.; Coquand, R.; Maffini-Alvaro, V.; Samson, M.-P.; Hartmann, J.-M.; Tosti, L.;

Casse, M.; Nguyen, V.-H.; Triozon, F.; Niquet, Y.-M.; Tabone, C.; Perreau, P.; Allain, F.; 
Vizioz, C.; Comboroure, C.; Aussenac, F.; Monfray, S.; Ghibaudo, G.; Boeuf, F.; De Salvo, B.; Faynot, O. VLSI Tech. Symp. 2013, T230-T231.

3. Ding, Y.; Zhou, Q.; Liu, B.; Gyanathan, A.; Yeo, Y.-C. IEEE T. Electron. Dev. 2014, 61, 1963-1971.

4. Cheng, R.; Ding, Y.; Koh, S.-M.; Yang, Y.; Bai, F.; Liu, B.; Yeo, Y.-C. IEEE T. Electron. Dev. 2014, 61, 2647-2655.

5. Nguyen, P.; Barraud, S.; Tabone, C.; Gaben, L.; Casse, M.; Glowacki, G.; Hartmann, J.M.; Samson, M.-P.; Maffini-Alvaro, V.; Vizioz, C.; Bernier, N.; Guedj, C.; Mounet, C.; Rozeau, O.; Toffoli, A.; Alain, F.; Delprat, D.; Nguyen, B.-Y.; Mazure', C.; Faynot, O.; Vinet, M. Tech. Digest IEEE Electron Devices Meet 2014, 16.2.1-16.2.4 


\section{Supporting Information 4: GIDL in the VM-FET}

Compared to single nanowire FET, the fabricated VM-FET can be vulnerable to gateinduced-drain-leakage (GIDL), which stem from the structure and the fabrication process of the VM-FET. In this study, the size of the gate buried through the trench hole pattern (Figure 2a in manuscript) is all equal regardless of the length of the exposed gate. When the length of the exposed gate is reduced, the S/D implanted region in buried gate is widen, resulting in the increase of gate-to-drain overlap region. In result, the GIDL is being increasingly severe as the gate length decreases, as shown in Figure S4 (a).

However, this problem can be improved via the revision of layout of further VM-FET. As shown in Figure S4 (b), gate-to-drain overlap region caused by the S/D implantation is remarkably reduced when the length of the buried gate become equal to that of the exposed gate. Such a configuration can be easily fabricated by modulating the size of the trench hole pattern in the layout. Considering sequential S/D implantation process, the formation of the gate spacer also is good way to improve the GIDL. In conclusion, using layout revision and typical silicon process, the GIDL can be reduced in the further VM-FET.

(a)

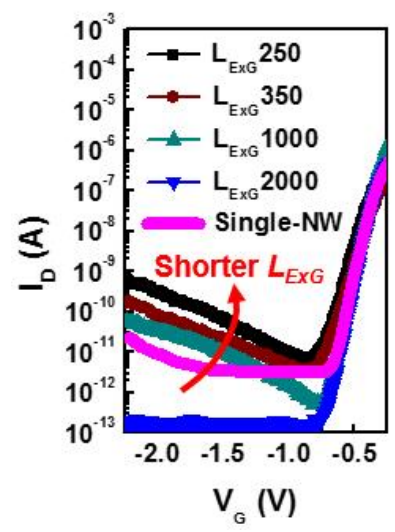

(b)

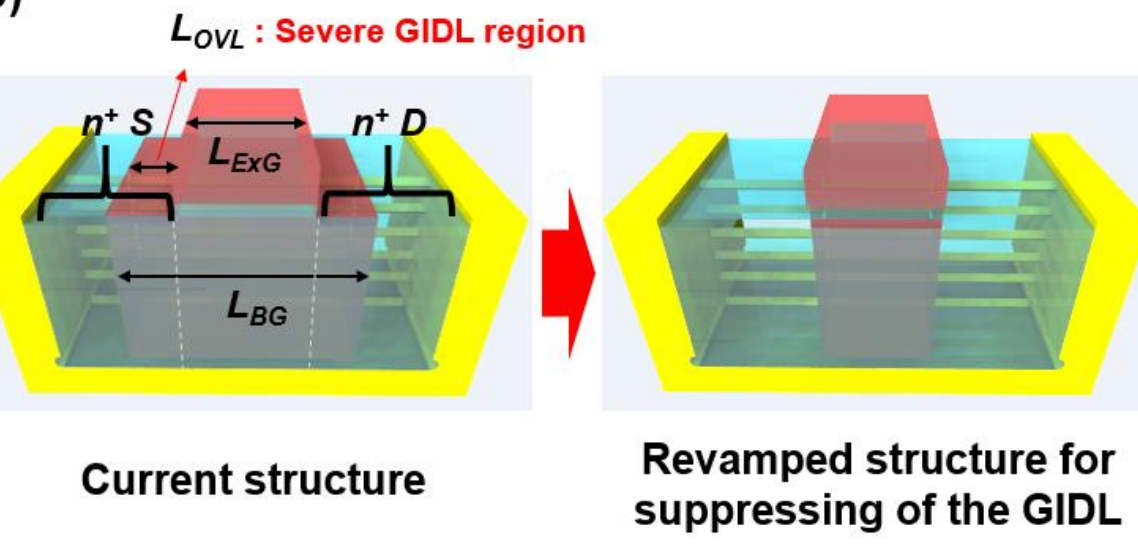


Figure S4. (a) GIDL of the VM-FET with various gate lengths and the comparison to the GIDL of the single nanowire FET. The longer $\boldsymbol{L}_{\boldsymbol{O} V \boldsymbol{L}}$, the larger GIDL. In the schematic, $\boldsymbol{L}_{\boldsymbol{E} \boldsymbol{x} \boldsymbol{G}}$ and $\boldsymbol{L}_{\boldsymbol{B} \boldsymbol{G}}$ represent a length of the exposed gate and buried gate, respectively. And $\boldsymbol{L}_{\boldsymbol{O} \boldsymbol{}} \boldsymbol{}$ is the overlap length of gate-to-S/D. (b) Structural modification of the VM-FET to reduce the GIDL. Such a revamped structure can easily be achieved through the layout revision. 


\section{Supporting Information 5: VM-FET with laterally parallel stacks of the}

\section{nanowire}

To maximize the current drivability, a design for the VM-FET with laterally parallel stacks of the nanowire is considered. Here, a provisional feasibility to fabricate the structure is discussed. Figure S5 (a) shows the structure. The laterally high-integration of the nanowire for the structure depend on photolithography rather than the ORADEP. As shown in Figure S5 (b), an initial process to form currently vertically integrated nanowire is the patterning of the hard mask. At this process, the dimension of the hard mask and space, i.e., pitch in Figure S5 (b), is determined by the photolithography process, thereby strongly influencing on the laterally parallel stacks of the nanowire. While the scaling down of the hard mask width is relatively easy by the use of the sacrificial oxidation or photoresist trimming process compared with the reduction of the spacing. Or iterative spacer patterning processes are useful to increase the packing density. ${ }^{1-3}$

The ORADEP would not be a severe obstacle for laterally parallel stacks of the nanowire for high packing density. In particular, it is expected that $\mathrm{C}_{4} \mathrm{~F}_{8}$ passivation would be done well with good step coverage even in the case of the narrow pitch due to the optimization of various adjustable factors such as the chuck bias, chamber temperature, and gas amount. In case of SF6 isotropic etch to form dense patterns, an incidence angle of the plasma-based ion is reduced, resulting in a decrease of the etch rate. This is advantageous for the controllability of the etching process. In view of the overall processes, reducing the $\mathrm{C}_{4} \mathrm{~F}_{8}$ gas amount and $\mathrm{SF}_{6}$ etch rate under the same pressure is effective for fabricating laterally parallel stacks of the nanowire with high density. If photolithography process permits the narrow pith of hard mask, the fabrication of the structure would be sufficiently possible by modulating the ORADEP condition. 
Due to the issue mentioned above, it is difficult to conclude the accurate pitch. Figure S5 (c) shows a result of the pilot test for the proposed structure under the KrF-based photolithography process, even though it is not a critical pitch of tens-of-nanometer. This result may demonstrate a provisional feasibility for the proposed structure with the critical pitch. It is expected that the feasibility of a critical pitch-based the structure would be considerably high with the aid of high-resolution photolithography.

(a)

(c)
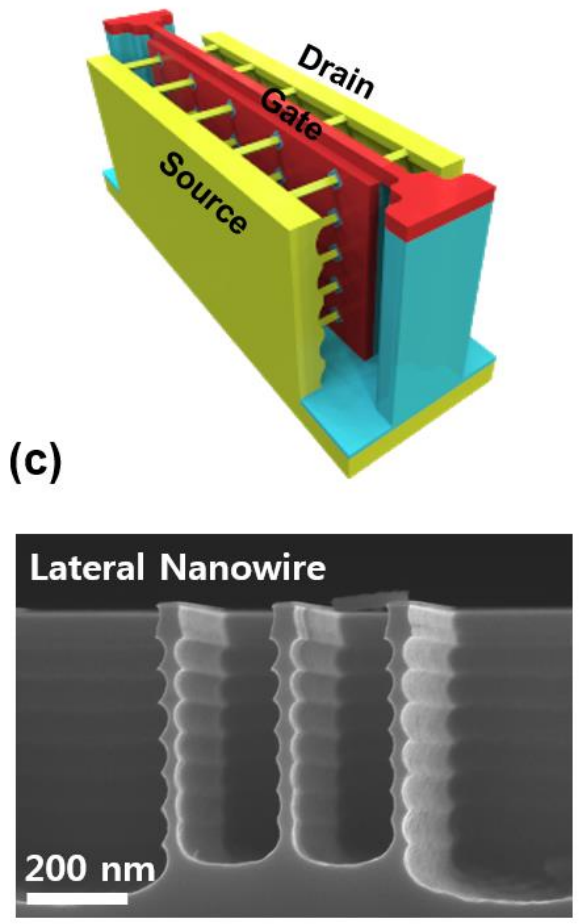

(b)

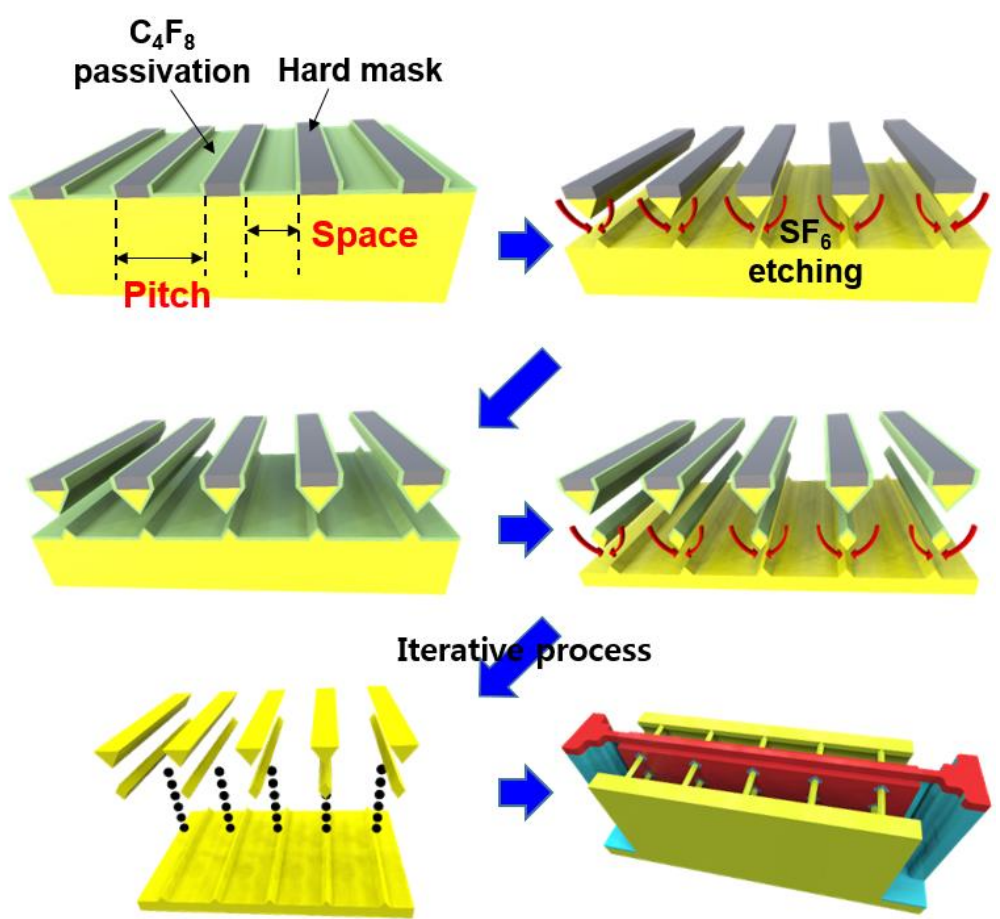

Figure S5. Schematic and pilot test for the proposed configuration of the VM-FET with multiple lateral channel. (a) Schematic of the proposed FET. (b) Fabrication process to form the laterally parallel stacks of the nanowire. (c) Result of the pilot test to show a provisional feasibility.

\section{Reference}

1. Choi, Y.-K.; King, T.-J.; Hu, C. IEEE Electr, Device L. 2002, 49, 436- 441. 
2. Choi, Y.-K.; Lee, J.-S.; Zhu J.; Somorjai, G. A.; Lee, L. P.; Bokor, J. J. Vac. Sci. Technol. B, 2003, $21,2951-2955$

3. Yeon, J.; Lee, Y. J.; Yoo, D. E.; Yoo, K. J.; Kim, J. S.; Lee, J.; Lee, J. O.; Choi, S.-J.; Yoon, G.-W.; Lee, D. W.; Lee, G. S.; Hwang, H. C.; Yoon, J.-B. Nano Lett. 2013, 13, 3978-3984. 